\title{
Einleitung zum Symposium über Michael HAMPE, Die dritte Aufklärung, Berlin, Nicolai Publishing, 2018
}

Ana Honnacker and Tullio Viola

\section{(2) OpenEdition}

\section{Journals}

Electronic version

URL: http://journals.openedition.org/ejpap/2163

DOI: 10.4000/ejpap.2163

ISSN: 2036-4091

\section{Publisher}

Associazione Pragma

\section{Electronic reference}

Ana Honnacker and Tullio Viola, « Einleitung zum Symposium über Michael HAMPE, Die dritte Aufklärung, Berlin, Nicolai Publishing, $2018 »$, European Journal of Pragmatism and American Philosophy [Online], XII-2 | 2020, Online since 14 December 2020, connection on 15 December 2020. URL : http:// journals.openedition.org/ejpap/2163; DOI : https://doi.org/10.4000/ejpap.2163

This text was automatically generated on 15 décembre 2020 .

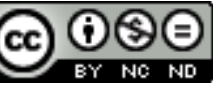

Author retains copyright and grants the European Journal of Pragmatism and American Philosophy right of first publication with the work simultaneously licensed under a Creative Commons AttributionNonCommercial-NoDerivatives 4.0 International License. 


\title{
Einleitung zum Symposium über Michael HAMPE, Die dritte Aufklärung, Berlin, Nicolai Publishing, 2018
}

\author{
Ana Honnacker and Tullio Viola
}

\section{AUTHOR'S NOTE}

Das Symposium wurde im Namen des German Pragmatism Network organisiert

1 Mit der Dritten Aufklärung, die zugleich titelgebend für den Gegenstand dieses Symposiums ist, nimmt der Zürcher Philosoph Michael Hampe eine Idee Hilary Putnams wieder auf. In seiner in Amsterdam gehaltene Spinoza-Vorlesung "The Three Enlightenments" hatte Putnam von drei Aufklärungsperioden in der Geschichte der Philosophie und der Kultur gesprochen. Die erste sei die in Athen mit Sokrates und Plato stattgefundene philosophische Revolution, die aus der Entdeckung des kritischen Denkens als "reflektiver Transzendenz" entsprang. Die zweite sei die europäische Aufklärung des 17. und 18. Jahrhunderts, die, gespeist aus der Theorie des Sozialvertrags und der Neufundierung der Naturwissenschaft, zu einem erneuerten Glauben an das kritische Vermögen des Individuums, selbst zu denken, fand. Die dritte Aufklärungszeit schließlich sei "one that hasn't happened yet, or hasn't at any rate fully happened, but one that I hope will happen, and one worth struggling for." Ihr Wegweiser sei John Dewey: Seine Kopplung von epistemischem Fallibilismus und antiskeptischer Haltung sowie seine Insistenz auf der Anwendung "of intelligence to problems" ermögliche eine demokratische Erweiterung und eine anti-rationalistische Vertiefung der klassischen Aufklärungsideale. ${ }^{1}$

2 Hampe folgt dem argumentativen Schema Putnams, gibt ihm jedoch einen noch vorsichtigeren und womöglich pessimistischeren Unterton: Eine dritte Aufklärung habe noch nicht einmal wirklich begonnen. Sie werde nur stattfinden, wenn epochale 
Änderungen in der Kultur eintreten, die unsere globale Gesellschaft durchdringt. Sie sei eine Aufgabe - eine dringende aber höchst ungewisse Aufgabe -, vor der wir stehen.

Das Wort Kultur kann tatsächlich als Schlüsselbegriff in Hampes Argumentation betrachtet werden. Ganz am Anfang seines Essays steht die These, dass die heute von mehreren Seiten diagnostizierte Krise der Demokratie eigentlich auf einer Krise und Erosion der aufgeklärten (politischen) Kultur beruht, die die Demokratie als Regierungsform fördert, mit dieser aber nicht in eins fällt (8f.). Hampe verweist darauf, dass sich die demokratische Regierungsform ebenso ohne aufgeklärte Kultur finden lässt wie ohne Marktwirtschaft und Achtung der Menschenrechte, die ebenfalls nicht notwendigerweise mit ihr einhergehen. Umgekehrt sei eine aufgeklärte Kultur nicht unbedingt an die Demokratie gebunden (obwohl wahrscheinlich nur letztere dafür sorgt, dass die aufgeklärte Kultur nicht zu einem elitären Produkt wird).

Hampe geht also von einem Primat der aufgeklärten Kultur aus, die der Schlüssel zu der Art und Weise ist, wie das politisch-gesellschaftliche Leben sich jeweils ausgestaltet: repressiv oder kritikfähig, intolerant oder tolerant, gewaltsam oder am diskursiven Austausch orientiert. Dementsprechend gelte es, den (gewissermaßen als Krise der Demokratie zu kurz beschriebenen) Krisenphänomenen dadurch $\mathrm{zu}$ begegnen, eine andere politische Kultur anzustreben, kurz: sich für eine Dritte Aufklärung einzusetzen. Genau dieser letzte Punkt wird von Yvonne Hütter-Almerigi und Dirk Jörke, die die beiden kritischen Diskussionsbeiträge des Symposiums verfasst haben, bestritten, und zwar mit dem Argument, dass die Existenz kultureller Defizite zu kurz greife, um die gefährlichsten politischen Phänomene der jüngeren Zeit - etwa den globalen Erfolg rechtspopulistischer Bewegungen - zu erklären. Das Bestehen sozialer oder wirtschaftlicher Ungleichheiten sei, so die Kritik, für das Verständnis der heutigen politischen Situation viel relevanter. Doch was heißt Kultur bei Hampe?

In seiner Replik an Hütter-Almerigi und Jörke macht Hampe sein Verständnis dieses Begriff explizit, indem er ihn mit dem Begriff der Lebensform gleichsetzt. Kultur sei die ganze Art und Weise, wie eine Gesellschaft lebt, denkt, und aufgrund derer - wie er bereits im Buch sagt - ihr "politische[s] Leben stattfindet" (10). Bei näherer Betrachtung jedoch ist die Lage komplizierter, spannungsreicher und in dieser Spannung möglicherweise auch produktiver. Denn bei Hampe findet sich neben dieser, an sich vollkommen plausiblen und gängigen Definition ein weiterer Zugang zum Kulturbegriff: Kultur ist bei ihm auch das Ergebnis von Bildung und das Produkt intellektuell ausgefeilter Aktivitäten wie Kunst, Wissenschaft und Philosophie. Es ist genau die Art und Weise, auf die er diese beiden Auffassungen zu verknüpfen vermag, die das Spezifikum von Hampes Kulturkritik ausmacht und ihn als aufklärerischen Denken definiert. Bildung ist eine "Bedingung für Kultur" (19); Philosophie und Kunst haben einen Einfluss auf die politische Kultur, auf die Ausgestaltung des politischen Lebens einer Gesellschaft und können dieses (ganz ohne billigen Fortschrittsoptimismus) verbessern.

6 Gewiss, seine Position setzt sich einer Kritik aus, die Dirk Jörke besonders zugespitzt artikuliert: Hampe drohe dem "scholastischen Trugschluss" (Bourdieu) anheimzufallen, indem er Bildung als Hauptmotor des Kulturwandels sieht; andere Strukturen und Dynamiken seien viel mächtiger als Erziehung oder intellektuelle Arbeit, um politische Lebensformen in neue Richtungen zu lenken. Man kann aber auch Hampes Vorschlag als ein Plädoyer dafür interpretieren, dieses aufklärerische Ideal nicht so sehr als eine soziologische These $\mathrm{zu}$ verstehen, sondern als eine $z \mathfrak{u}$ realisierende Aufgabe. Mit 
expliziter Referenz auf Kant definiert er Aufklärung als Mündigkeit (25-7), Illusionslosigkeit, und Autonomie des Lebensgestaltung (23-5). Diese Ideale sind heute von besonderem Belang, um eben die Rolle der Bildung und des Wissens angesichts gegenwärtiger Herausforderungen - fake news sind hier das prominenteste Beispiel - zu verteidigen.

7 Doch ist Hampes Definition der dritten Aufklärung deutlich reicher als dieses allgemeine Plädoyer fürs sapere aude! Drei Ideen lassen sich insbesondere hervorheben.

Erstens ist das Ziel der Autonomie bzw. des Selbstdenkens nur dann zu erreichen, wenn es durch eine Pluralität von "Kultur-" (65) und "Wahrheitspraktiken" (34) verfolgt wird, kraft derer die sozialen Akteure zu objektiver - obwohl immer revidierbarer - Kenntnis gelangen können. Diese Idee ist möglicherweise die originellste und fruchtbarste in Hampes Ansatz: Die Debatte zwischen Relativismus und Universalismus kann man pragmatistisch einklammern, indem man auf die Kultivierung von einzelnen Praktiken in der Erforschung sozialer Probleme fokussiert. Zudem ist Hampes Fokussierung auf ganz konkrete Wahrheitspraktiken auch darauf abzielend, das "mangelnde Bewusstsein für die normativen Auswirkungen des technischen Wandels, vor allem auf die Normen des Wissen" (15), zu kompensieren.

Hampe ist weiter davon überzeugt, dass wirkliche Autonomie des Denkens und der Lebensgestaltung nur auf Basis einer bestimmten Auffassung der Geschichte erreicht werden kann, die den gemischten Charakters des menschlichen Fortschritts (76-9) hervorhebt. Es gibt kein Schicksal in der Geschichte: Die Folgen menschlicher Handlungen entstehen aus einer Kombination von Zufall und Notwendigkeit. Diese These ist Hampe wichtig, um einerseits der naiven Illusion über die Linearität des Fortschritts entgegenzuwirken, andererseits aber den Verdacht abzulehnen, dass das individuelle Handeln keinen Einfluss auf historische Prozesse habe. Es ist zentral für das Gelingen der Aufklärung, dass "die Menschen sich als Teilnehmer ihrer Welt verstehen" (82).

Diese Bewusstseinsbildung darf dabei aber keine individuelle (oder partikularistischnationale) bleiben. Vielmehr verknüpft Hampe, drittens, Aufklärung eng mit dem Begriff der Solidarität und des Vertrauens. Wahrheitspraktiken sind eben nicht nur eine rein epistemische, sondern zugleich auch ethische Angelegenheit. Um gemeinsame Ziele zu verfolgen, das Leben aller zu verbessern, gilt es, "eine Basis geteilter Wahrheiten" (31) zu etablieren. Eine zentrale Rolle spielt dabei für Hampe nicht zuletzt der interkulturelle Dialog (20). Dieser dient einerseits der Vermeidung von Krieg und Gewalt, andererseits ist er besonders relevant in einer Zeit, in der jede Kultur, die Einfluss aufs politische Leben üben kann, sich als global verstehen sollte. Gegenseitiges Verständnis und gemeinsame Zielvorstellungen sind dabei, entgegen einem anthropologischen Essentialismus, Ergebnis einer kollektiven Anstrengung (21f.). An dieser, durch interkulturelle Bildung unterstützten Anstrengung hänge es, ob es der Menschheit gelinge, "sich durch Steigerung des kollektiven Bewusstseins zum Subjekt ihrer eigenen Geschichte" zu machen, und damit zu einem "handlungsfähigen Subjekt zusammen[zu]wachsen" oder aber "zivilisatorisch unter[zu]gehen" (20).

11 Den Versuch, Grausamkeit zu vermeiden, sieht Hampe schließlich als "Kern oder Kristallisationspunkt" der Aufklärung, der sich in der Bekämpfung von Unterdrückung, Illusionen und Ungerechtigkeiten fortsetzt (39f.). Hier kommt der Dialog mit einem weiteren Pragmatisten zutage, nämlich Richard Rorty. Und wieder spielt Bildung eine zentrale Rolle, weil sie die Widerstandsfähigkeit des Menschen gegen Täuschungen und 
Unwahrheiten gewährleistet, die in die Repression und Gewalt führen. Diese Errungenschaft der aufgeklärten Lebensform aber ist, wie Hampe rundheraus zugibt, eine prekäre: Grausamkeit und Gewalt können immer wieder über sie hereinbrechen, von außen, aber auch, weil sie, sofern sie auf geeigneten situativen Nährboden fallen, eine Neigung des Menschen $\mathrm{zu}$ sein scheinen, die seinem "Bedürfnis nach widerständiger Wirklichkeit" (48), einem Streben nach Intensität (61-4) entspringen. Diese Disposition realisiert sich aber nur unter bestimmten sozialen Bedingungen, sie ist sozusagen ein "Aggregatzustand” des Menschen, eine seiner Möglichkeiten. Die Bildungs- und Kultivierungsanstrengungen der aufklärerischen Bewegung sind daher eben genau das: Anstrengungen, deren Praktiken permanent neu angeeignet und vermittelt werden müssen.

Ein weiteres wichtiges Hilfsmittel in der Vermeidung der Grausamkeit sieht Hampe in der Kultivierung ästhetischer Erfahrung. Die Rolle der Kunst und Kultur tritt damit noch einmal in den Vordergrund: Sie kann das menschliche Bedürfnis nach intensiver Erfahrung in einer Art und Weise befriedigen, die mit einer demokratischen Gesellschaft durchaus kompatibel ist. Bildung kommt damit eine tragende Rolle bei der "Kriegsvermeidung" (64) zu. Dabei bleibt aber eine Frage offen, die Hütter-Almerigi anspricht: Ist eine solche Auseinandersetzung mit dem Problem der Gewalt als normative Achse eines erneuerten Aufklärungsideal hinreichend? Wird nicht ein nicht unbeträchtlicher Teil der Gewalt bzw. Unterdrückung ohne Grausamkeit, also quasi "indirekt" oder auf subtilere Weise ausgeübt?

Die dritte Aufklärung, soviel wird deutlich, bleibt nicht nur ein permanentes Projekt, dass der gemeinsamen Anstrengung bedarf. Sie ist darüber hinaus darauf angewiesen, die konkreten Problemkonstellationen und -dynamiken genau $\mathrm{zu}$ analysieren, um tatsächlich nicht nur $\mathrm{zu}$ einem Bildungsideal, sondern auch $\mathrm{zu}$ einer praktischen Lebensform zu werden.

\section{NOTES}

1. Hilary Putnam, “The Three Enlightenments” (2001), in Id., Ethics without Ontology, Cambridge, Ma./London, Harvard University Press, 2004, 89-108.

\section{AUTHORS}

\section{ANA HONNACKER}

Universität Hildesheim

post[at]ana-honnacker.de 
TULLIO VIOLA

Universität Erfurt

tullio.viola[at]gmail.com 